\title{
The role of subjective frequency in language switching: An ERP investigation using masked priming
}

\author{
Krysta Chauncey • Jonathan Grainger • \\ Phillip J. Holcomb
}

Published online: 4 November 2010

(C) The Psychonomic Society 2010

\begin{abstract}
Two experiments examined the nature of language-switching effects in a priming paradigm with event-related brain potential (ERP) recordings. primes and targets were always unrelated words but could be either from the same or different languages (Experiment 1) or from the same or a different frequency range (Experiment 2). Effects of switching language across prime and target differed as a function of the direction of the switch and prime duration in Experiment 1. Effects tended to be stronger with $100-\mathrm{ms}$ prime durations than with 50-ms durations, and the expected pattern of greater negativity in the switch condition appeared earlier when primes were in L1 and targets in L2 than vice versa. Experiment 2 examined whether these language-switching effects could be due to differences in the subjective frequency of words in a bilingual's two languages, by testing a frequency-switching manipulation within the L1. Effects of frequency switching were evident in the ERP waveforms, but the pattern did not resemble the languageswitching effects, therefore suggesting that different mechanisms are at play.
\end{abstract}

Keywords Language switching · Bilingualism $\cdot$ Masked priming $\cdot$ ERPs

K. Chauncey $\cdot$ P. J. Holcomb

Tufts University,

Medford, USA

J. Grainger

CNRS \& Aix-Marseille University,

Marseille, France

J. Grainger $(\bowtie)$

Laboratoire de Psychologie Cognitive, Université de Provence,

3 pl. Victor Hugo,

13331 Marseille, France

e-mail: jonathan.grainger@univ-provence.fr
Speakers comfortable in several languages often use more than one language within a discourse or even within a sentence, particularly when other bilinguals are present. Although such language switching or code switching may have social or communicative benefits, it is generally thought to come with a cognitive cost: Both comprehension and production are slower with mixed-language stimuli, and in comprehension, this effect is even more marked when the input language changes unpredictably (Dalrymple-Alford, 1985; Grainger \& Beauvillain, 1987; MacNamara \& Kushnir, 1972; Meuter \& Allport, 1999; Orfanidou \& Sumner, 2005; Soares \& Grosjean, 1984; Thomas \& Allport, 2000; von Studnitz \& Green, 1997; see Costa \& Santesteban, 2004 , for studies of language-switching effects in production). The present study provides a further investigation of the nature of such processing costs in language comprehension.

As has been noted by Chauncey, Grainger, and Holcomb (2008), a key question guiding research on language switching is whether switch costs are caused by a general task control mechanism or are the result of processes specific to the control of language activation in bilinguals. In one prominent model of bilingual language comprehension, the bilingual interactive activation (BIA) model (Grainger \& Dijkstra, 1992; van Heuven, Dijkstra, \& Grainger, 1998), language switch costs are caused by a mechanism controlling the relative activation of lexical representations in each language-so-called language nodes. In the original BIA model, the language node mechanism has a direct influence on the relative activation level of lexical representations in each language, contrary to later developments of this model (the BIA + model; Dijkstra \& van Heuven, 2002). According to the original BIA model, processing costs following a language switch are the result of continuing 
top-down inhibition from the inappropriate language node toward the lexical representations of the target language.

However, perhaps the most popular account of switch costs is that they are almost exclusively the result of executive control factors and, thus, external to the language system and related, instead, to how participants control their decisions and responses in a laboratory task (Dijkstra \& van Heuven, 2002; Green, 1998; Thomas \& Allport, 2000). In Green's inhibitory control (IC) model, the concept of task schemas plays a central role in accounting for switch costs in the comprehension and production of language in bilinguals. Task schemas are part of a general task control system and are used in the bilingual situation to link the output of lexical processing to a specific behavioral response. They are mutually inhibitory, such that, after using task schema A, it is harder to apply task schema B than to repeat task schema A. Since producing language always requires a behavioral response, the IC model has found interesting applications in this area (see, e.g., Finkbeiner, Gollan, \& Caramazza, 2006). Concerning language comprehension, bilinguals do not need to be informed in advance of the language of the incoming stimulus in order to understand it; hence, switch costs in comprehension do not necessarily reflect the influence of task schemas. However, much of the focus of laboratory research on language switching in recent years has been on situations involving a different response associated with each language (e.g., respond "yes" if the word is in a specific language and "no" otherwise)-hence, artificially exaggerating the possible influence of task schemas.

One early study of language switching initiated a different approach. Grainger and Beauvillain (1987) found switch costs in a generalized lexical decision task (participants responded "word" if the target was a word, regardless of language, and "nonword" otherwise). In this task, therefore, the language affiliation of the stimulus has no consequences for the required response. Such language information could therefore, in principle, be ignored, but Grainger and Beauvillain demonstrated that this information is not ignored. Lexical decision times to words in one language were slower when the preceding trial was in a different language, as compared with a preceding stimulus from the same language, and these switch costs tended to be larger for second-language (L2) targets than for first-language (L1) targets. This basic result was later replicated by von Studnitz and Green (1997) and Thomas and Allport (2000) and provides support for the hypothesis that at least part of languageswitching effects in comprehension are being driven by a process that is independent of more general decisionrelated or task-related mechanisms.

\section{Language switching and event-related brain potentials}

Event-related brain potentials (ERPs) offer the possibility of measuring language-switching effects in language comprehension in conditions in which participants are silently reading for meaning. A further advantage to using ERPs to study language processing in general and language switching effects in particular is the gain in sensitivity to processing as it unfolds over time. This provides key information about the relative timing of different effects and can also allow influences of a given variable to be detected in conditions in which the final product of processing (i.e., an overt response in a given task) lacks sensitivity. In one of the first ERP studies to examine language-switching effects, Alvarez, Holcomb, and Grainger (2003) measured ERPs to single words presented in lists containing a random mixture of words from both of the participants' languages. Participants simply had to press a response button whenever they saw an animal name in either language, which appeared on approximately $10 \%$ of the trials. The critical trials, which did not require a behavioral response and to which ERPs were recorded, were nonanimal words. They found that the N400 ERP component was more negativegoing to L2 words following anL1 word than to L2-L2 sequences of otherwise unrelated words (see Moreno, Federmeier, \& Kutas, 2002, for a similar result obtained with mixed-language sentences). On the other hand, there was no evidence of switching effects on ERPs generated to L1 targets. One possibility, given the relatively long interstimulus interval in the Alvarez et al. study is that participants had sufficient time to translate the word on trial $n$-1 before processing words on trial $n$, and given their relatively low proficiency level in Spanish (L2), they might well have translated the L2 words. Therefore, if an L2 item is translated into L1 and then an L1 item appears on the following trial, less of a switch cost may occur, since the L1 lexicon is already engaged in processing the word on the previous trial. This interpretation of the results of Alvarez et al. (2003) fits with the proposed differential strength of connectivity between the lexical representations of $\mathrm{L} 1$ and L2 translation equivalents (stronger in the direction of $\mathrm{L} 2$ to L1) in the revised hierarchical model of Kroll and Stewart (1994).

In order to circumvent such translation strategies, Chauncey et al. (2008) examined the effects of language switching using a masked priming paradigm with ERP recordings. Because all targets in a single block were in one language, whereas primes could be in either language, participants were unaware that language switches (across prime and target) were occurring on some trials, and thus it was reasoned that participants would not be able to use conscious predictive or translation strategies. Chauncey et al. found language-switching effects on two ERP compo- 
nents - the N250 and N400 - that differed as a function of the direction of the switch, but with switches always being associated with greater negativities. L2 targets showed switch effects mainly on the early component (N250), whereas for L1 targets, most of the switch effects appeared on the later N400. Extensive research examining priming effects in monolinguals (e.g., Holcomb \& Grainger, 2006, 2007; see Grainger \& Holcomb, 2009, for a review) has suggested that the $\mathrm{N} 250$ component reflects the mapping of prelexical form representations (letters and letter clusters) onto whole-word form representations, whereas the N400 reflects the subsequent mapping of whole-word form representations onto semantics. In both cases, an increased negativity is generally interpreted as reflecting increased difficulty in processing the target word. Other examples of how increased negativity in the ERP waveforms is interpreted as reflecting increased difficulty in target word processing can be found in Grainger and Holcomb (2009).

Therefore, the results of Chauncey et al. (2008) suggest that language switches incur processing costs, the timing of which depends on the direction of the switch. Within the framework of the BIA model (Grainger \& Dijkstra, 1992; van Heuven et al., 1998), the different timing of the switch costs as a function of switch direction would reflect the different processing speed of L1 and L2 words. In the BIA model, language-switching effects are the result of automatic top-down modulation of activation of lexical representations as a function of language. In the priming situation tested by Chauncey et al., a prime word automatically activates its corresponding language node, which then inhibits or partially inhibits the representations of all the words in the other language (van Heuven et al., 1998). When the prime and target are from the same language, there is no inhibition on the target word. However, when the prime is from a different language, the target word representation receives inhibition from the language node activated by the prime word, and target processing is perturbed. L1 primes are processed faster than L2 primes and would, therefore, have an earlier and larger impact on the processing of L2 target words than L2 primes would on the processing of L1 target words. This would arise by faster activation of language nodes from L1 prime words and, therefore, faster acting inhibition on L2 targets. A strong prediction of the BIA model is that such fast-acting exogenous languageswitching effects exist and should be observable in the kind of priming paradigm used by Chauncey et al. (see Grainger, Midgley, \& Holcomb, in press, for further discussion). Chauncey et al. provided some initial evidence in support of this prediction, and the present study provides a further test of this key prediction of the BIA model.

\section{The present study}

Following Chauncey et al. (2008), the present study used a combination of priming methodology and ERPs in order to minimize task-related influences on language switching and to focus on stimulus-driven (exogenous) languageswitching effects. Chauncey et al. found a different pattern of language-switching effects as a function of the direction of the switch (L1-L2 switch effect appearing earlier than the L2-L1 effect), which might have been due to the relatively low proficiency of the participants in their L2. As was argued above, slower processing of prime words in the less fluent L2 could be the cause of the later appearance of language-switching effects from L2 to L1. One problem with this account is that Chauncey et al. did not find a change in the pattern of languageswitching effects when they increased the prime duration from 50 to $100 \mathrm{~ms}$. This might be because the extra prime duration was not sufficient to overcome the limitations imposed by the relatively low proficiency of the participants in their L2. Therefore, Experiment 1 in the present study was a direct replication of the Chauncey et al. study, but testing participants with a higher proficiency level in L2.

The second aim of the present study was to test one possible interpretation of the early language-switching effects seen on the N250 ERP component with L1 primes and L2 targets in the Chauncey et al. (2008) study. Specifically, we tested whether it might be the higher subjective frequency of the L1 prime word, as compared with an L2 prime word, that was causing the modulation of early ERPs. To do this, Experiment 2 tested for withinlanguage frequency-switching effects in bilingual participants' L1 by orthogonally varying prime and target word frequency. If the pattern of language-switching effects is partly due to differences in subjective frequency between L1 and L2 words, the within-language-switching manipulation should generate a similar pattern.

\section{Experiment 1}

Method

\section{Participants}

Forty participants ( 7 males; $18-22$ years of age, mean age $=$ 20.7) with an average immersive exposure to French of 5.9 months, in addition to seven collegiate French courses, were recruited from Tufts University and compensated for their time. All the participants had normal or corrected-tonormal visual acuity, with no history of neurological insult or language disability. 
Participants' knowledge of and skill in French was measured by a questionnaire about their language abilities and history, enumerating their experience (academic or other) with the French and English languages, in which they evaluated their reading and speaking skills in both languages (Table 1). The questionnaires were used to screen participants for the desired level of fluency. Participants indicated that they currently used French for an average of $7.3 \%$ of their day. So, although these participants were fluent in French, they were not balanced bilinguals. The self-evaluation ratings (7-point scale) for language skills in L2 (see Table 1) were significantly higher $(p<.01)$ for the present group of participants, for those tested by Chauncey et al. (2008); the corresponding values for the participants in the Chauncey et al. (2008) study for reading, speaking, and comprehension in L2 were $3.7,3.1$, and 3.1, respectively).

\section{Apparatus}

Electroencephalograms were collected using 32-channel caps (Electro-cap International), in which tin electrodes were arranged using the standard International 10-20 system (see Fig. 1). In addition to scalp electrodes, two electrodes were placed behind the ears on the left (A1) and right (A2) mastoid bones, with the A1 site used as an online reference for all other electrodes and the A2 site used to evaluate differential mastoid activity (there was none detected). A final two electrodes were placed directly below the left eye (to monitor for vertical eye movements and blinks) and to the right of the right eye (to monitor for horizontal eye movements. Impedance was measured at less than $2 \mathrm{k} \wedge$ for scalp and mastoid electrode sites and less than $10 \mathrm{k} \wedge$ for both eye channels. The EEG was amplified using an SA Bioamplifier (SA Instruments, San Diego, CA) operating on a pass band of $0.01-40 \mathrm{~Hz}$. The digitizing computer continuously sampled the EEG at a rate of $200 \mathrm{~Hz}$, and the stimulus computer simultaneously issued stimuli to the participant's monitor and transmitted codes indicated types of stimuli or behavior to the digitizing computer.

Table 1 Self-rating on a 7-point Scale for Reading, Speaking, and Oral Comprehension in their L1 and L2 of the Participants Tested in Experiment 1

\begin{tabular}{llll}
\hline & Reading & Speaking & Comprehension \\
\hline English (L1) & 7 & 7 & 7 \\
French (L2) & 5.38 & 5.36 & 5.64 \\
\hline
\end{tabular}

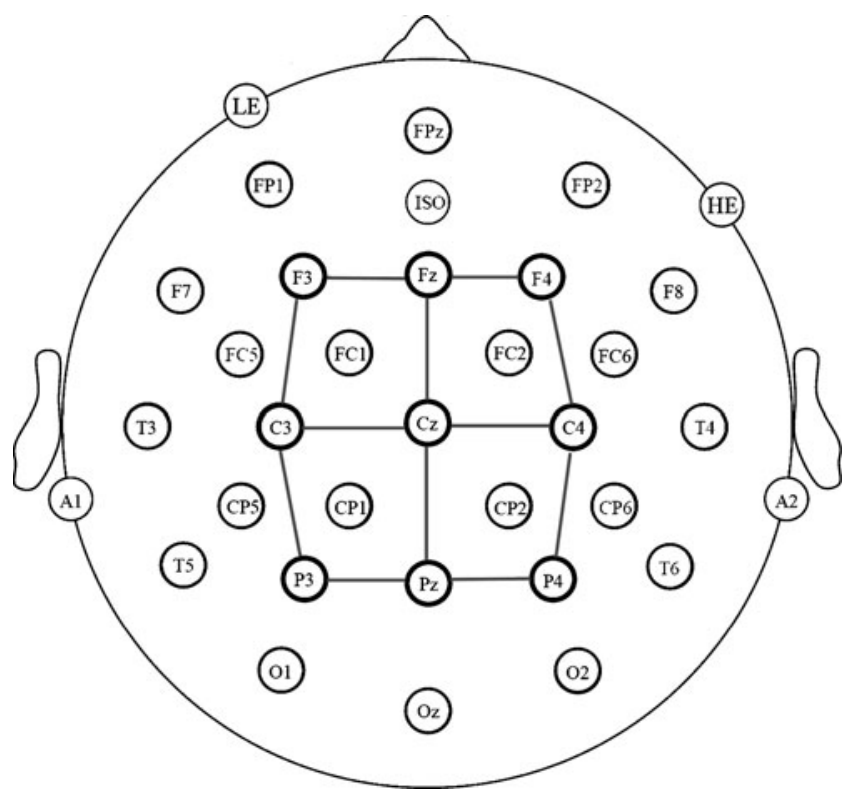

Fig. 1 Electrode montage and sites analyzed in ANOVAs of ERP data in both experiments

\section{Design and stimuli}

The design and stimuli were the same as those in the Chauncey et al. (2008) study. The stimuli for Experiment 1 consisted of prime-target pairs formed of completely unrelated words that were either in the same language or from different languages. All the words were between four and six letters and were selected to be as language-specific as possible - in particular, excluding close cognates. This was done in order to minimize confusion over which language was being presented within a given trial. Words with diacritics were also excluded in order to prevent the use of nonlexical cues. Translations of words used in one language were specifically excluded from the set of possible stimuli in the other language.

Target language was blocked, and all the participants were tested in both blocks, with the order of blocks counterbalanced across participants. In each language block, the language of the prime word was manipulated such that the prime could be in the same language as the target or in the other language. Two lists were constructed for each language block, so that overall, each target word appeared in both the same-language and differentlanguage prime conditions across participants, while avoiding stimulus repetition within participants. Primes were displayed in lowercase, and targets in uppercase. A random 20 of the participants were assigned to the shortprime-duration group (50-ms primes) and the remaining 20 were assigned to the long-prime-duration (100-ms) group. 
Each trial consisted of a 200-ms forward-patterned mask, a 50- or 100-ms presentation of the prime followed by a 10 -ms backward mask, a 300 -ms presentation of the target, and a $900-\mathrm{ms}$ blank interval and a 2,000 -ms endof-trial message, during which participants were instructed to blink if they needed to (see Fig. 2 for a schema of a typical trial). The next trial began $500 \mathrm{~ms}$ later.

\section{Procedure}

To ensure participant attention, as well as passive reading for meaning, a go/no-go semantic categorization task was imposed. Participants were asked to press a button whenever they saw an animal word. In each language block, $15 \%$ of the trials contained an animal word, half in the prime position and half in the target position. Animal words in the target position and the prime position were always in the same language as the other target words in the block. Including probes in the prime position provided an estimate of the level of visibility of prime stimuli.

After completing informed consent, language history, and handedness forms, participants were fitted with an electrode cap and were seated in a comfortable armchair in a sound-attenuated room with dimmed lighting. Before each experiment, participants were presented with a practice block to familiarize them with the trial sequence and the probe task in the target language. During the course of the experiment, the stimulus monitor was placed approximately $60 \mathrm{in}$. in front of the participants' chair. There were two breaks within each of the two experimental blocks, the length of which was determined by the participants. Words were presented centered horizontally and vertically on the participants' monitor, and each experimental block began when participants pressed a button to indicate their readiness. Each block typically lasted $30-35 \mathrm{~min}$, depending on the length of the breaks taken by the participants.

Analysis

ERPs were time-locked to the onset of the prime, but the 100 -ms pretarget period was used as a baseline. On the basis of visual analysis of the grand average waveforms and conventional windows for identifying ERP components in masked-priming experiments (e.g., Holcomb \& Grainger, 2006), mean amplitudes in three windows were measured: 200-300, 300-400, and 400-500 ms posttarget. Because direct comparisons of ERPs in different languages is of questionable validity, the data from the two target languages were analyzed separately and treated as two different subexperiments. In each subexperiment, a multifactor mixed design was used with each of the three mean amplitude measures and included a between-subjects factor of prime duration (50 vs. $100 \mathrm{~ms}$ ) and a within-subjects factor of language switch (within language vs. between language). Distribution variables of anterior-posterior (frontal vs. central vs. parietal) and laterality (left vs. center vs. right) were also included, to indicate each of the nine electrode sites included in the analysis (see Fig. 1, circled sites for the layout of the electrode locations included). The Geisser-Greenhouse correction was used with the two distributional variables.
Fig. 2 A typical trial in Experiment 1

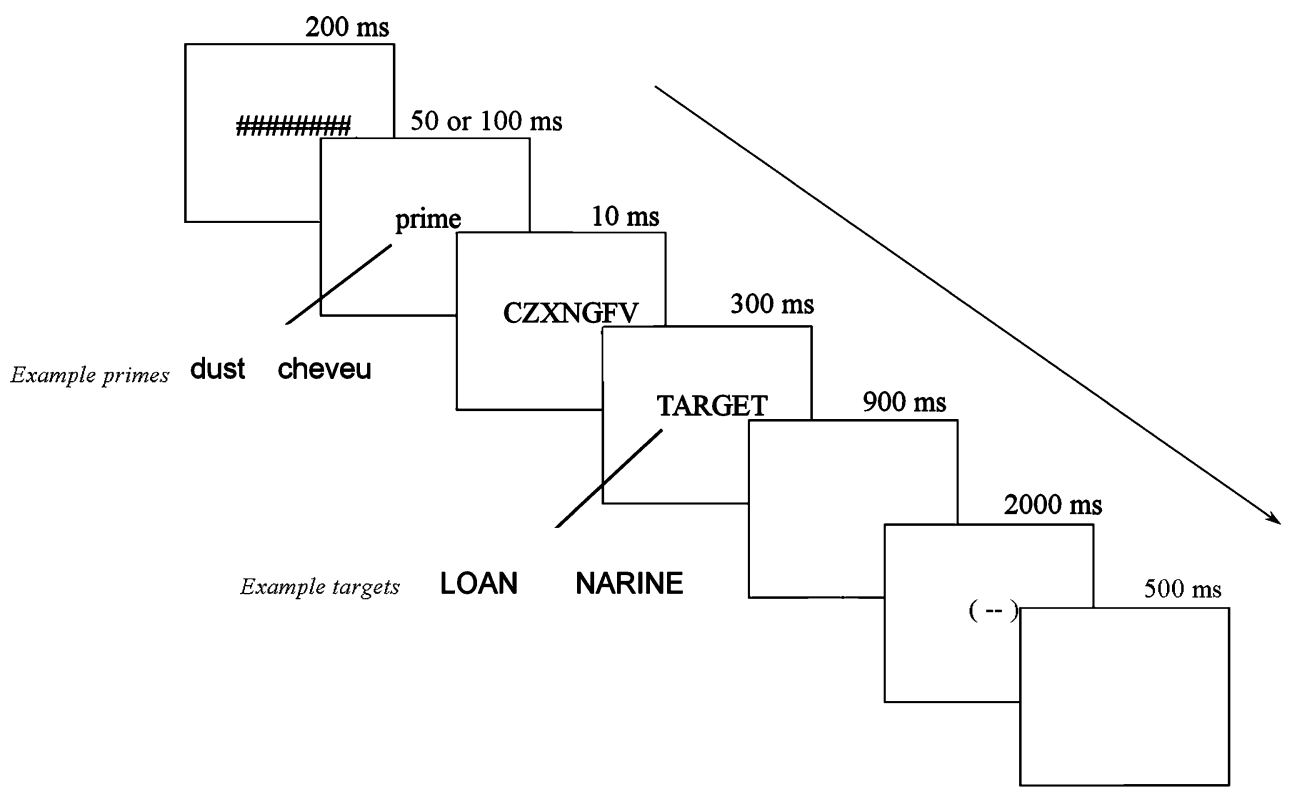




\section{Results}

\section{L1 target blocks}

Plotted in Fig. 3a $(50 \mathrm{~ms})$ and $\mathrm{b}(100 \mathrm{~ms})$ are the ERPs to L1 (English) targets preceded by primes in either L1 (solid) or L2 (dashed). Plotted in Fig. 4a (top row) are the voltage maps derived by subtracting L1 targets preceded by L2 primes from L1 targets preceded by L1 primes, averaged across both prime durations. Remember that these plots reflect an amalgamation of activity from the forward mask, the prime, the backward mask, and the target, all of which onset within a period of $260 \mathrm{~ms}$. Figs. 3 and 5 show a series of deflections in the ERP waveforms generated during target word processing that differ as a function of target language, prime language, and prime duration.

\section{$200-300 \mathrm{~ms}$}

There were main effects of prime duration, $F(1,38)=4.13$, $p=.0493)$ and language switch, $F(1,38)=11.68, p=.0015$, in this first measurement epoch. Targets following the 100ms primes produced greater negativity than did those following 50-ms primes, and within-language targets produced greater negativity than did between-language targets (a reversed-switching effect that was visible only at the 100ms prime duration; see Fig. 3). a
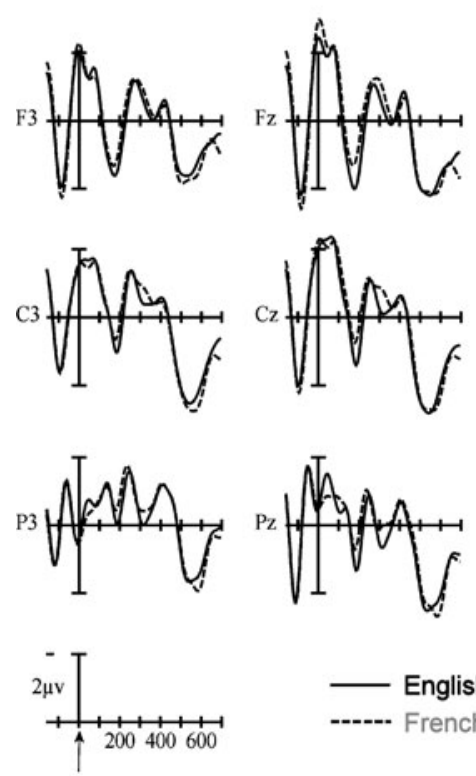

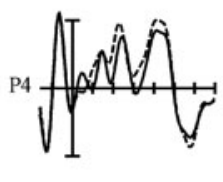

English Targets
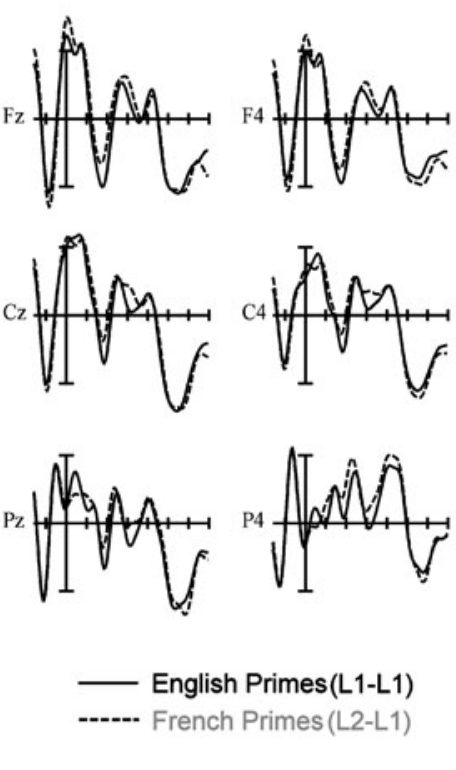

Fig. 3 a Target ERPs from selected sites in Experiment 1 in the 50ms prime condition, showing L1 (English) targets preceded by either L1 (English) primes (solid) or L2 (French) primes (dashed). The vertical calibration bar and the arrow on the time legend marks the

\section{$300-400 \mathrm{~ms}$}

In the middle time window, there were again main effects of prime duration, $F(1,38=4.76, p=.0353$, and language switch, $F(1,38)=20.86, p=.0001$. The 100 -ms prime condition continued to produce more negative-going potentials than did the 50-ms prime condition, but now the within-language targets produced greater negativity than did the between-language targets. The language-switching effect tended to be larger at central and frontal electrode sites [anterior-posterior $\times$ language switch interaction, $F(2$, 76) $=4.33, p=.034]$.

\section{$400-500 \mathrm{~ms}$}

In this final measurement epoch, there was a main effect of language switch, $F(1,38)=4.92, p=.0326$, with betweenlanguage targets producing more negative-going ERPs than did within-language targets.

\section{L2 target blocks}

Plotted in Fig. 5a are the ERPs to L2 (French) targets preceded by primes in either L2 (solid) or L1 (dashed) for the 50-ms prime duration condition, and in Fig. 5b are the comparable ERPs in the 100-ms prime duration condition. Plotted in Fig. 4a (bottom row) are the voltage maps b
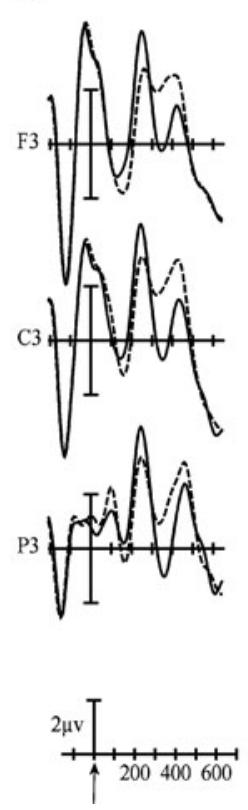

point of target onset. Negative values plotted upward and $x$-axis scale in milliseconds in this and all the following figures. b Target ERPs in the 100-ms prime duration condition; all else as in Fig. 3a 
a L1
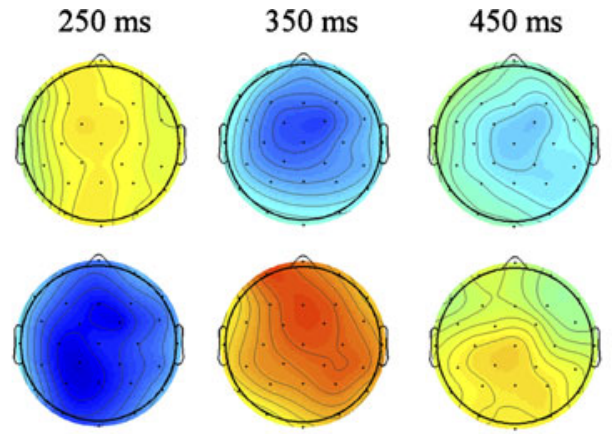

b
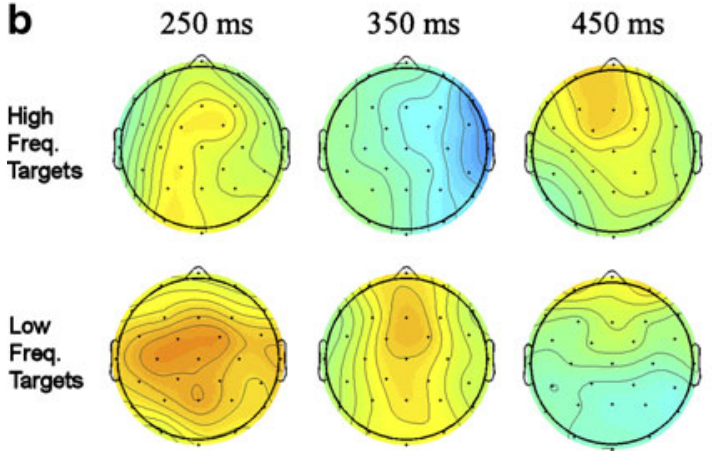

Fig. 4 a Voltage maps of difference waves (between-language trials minus within-language trials) at three latencies in Experiment 1 for L1 targets in the top row and L2 targets in the bottom row, averaged across the two prime durations. b Voltage maps of difference waves (switch trials minus nonswitch trials) in Experiment 2 for highfrequency targets in the top row and low-frequency targets in the bottom row a

French Targets
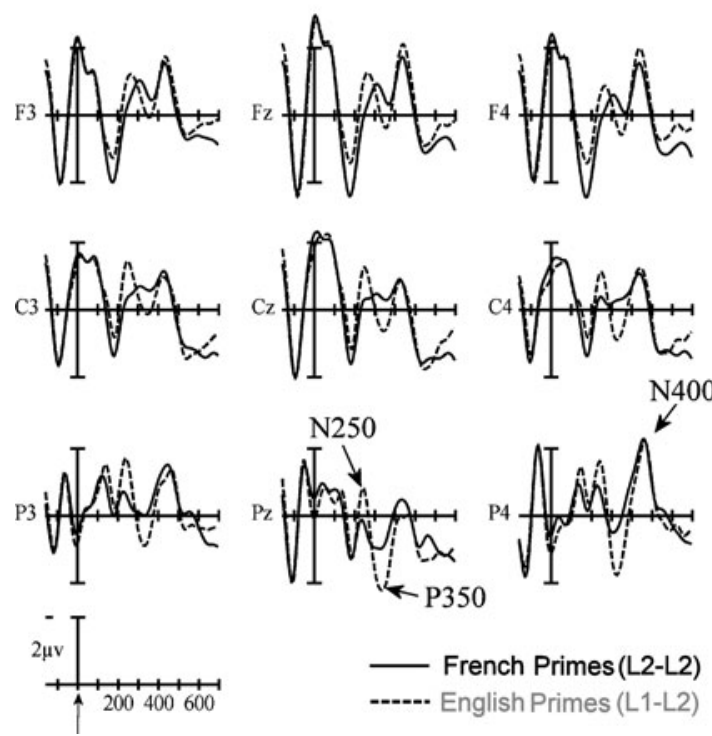

Fig. 5 a Target ERPs at selected sites in the 50-ms condition of Experiment 1, showing L2 (French) targets preceded by L2 (French) primes (solid) over-plotted with L2 (French) targets preceded by L1 derived by subtracting $\mathrm{L} 2$ targets preceded by $\mathrm{L} 1$ primes from L2 targets preceded by L2 primes, averaged across the two prime durations.

\section{$200-300 \mathrm{~ms}$}

In the first epoch, there were again main effects of prime duration, $F(1,38)=7.13, p=.011$, and language switch, $F$ $(1,38)=30.87, p=.00001$, with the 100 -ms prime duration condition producing more negative-going target waves than did the 50-ms prime duration condition and the betweenlanguage trials producing more negativity than did the within-language trials. Moreover, the effect of language switch tended to be larger for the $100-\mathrm{ms}$ prime duration condition than for the 50-ms condition [language switch $\times$ prime duration interaction, $F(1,38)=6.75, p=.0133]$.

\section{$300-400 \mathrm{~ms}$}

In this epoch, between-language targets were now more positive-going than within-language targets [main effect of language switch, $F(1,38)=14.25, p=.00005]$.

\section{$400-500 \mathrm{~ms}$}

In this last epoch, between-language trials produced a significantly more negative-going response than did withinlanguage trials, especially at more anterior sites [language switch $\times$ anterior-posterior interaction, $F(2,38)=4.39, p=$
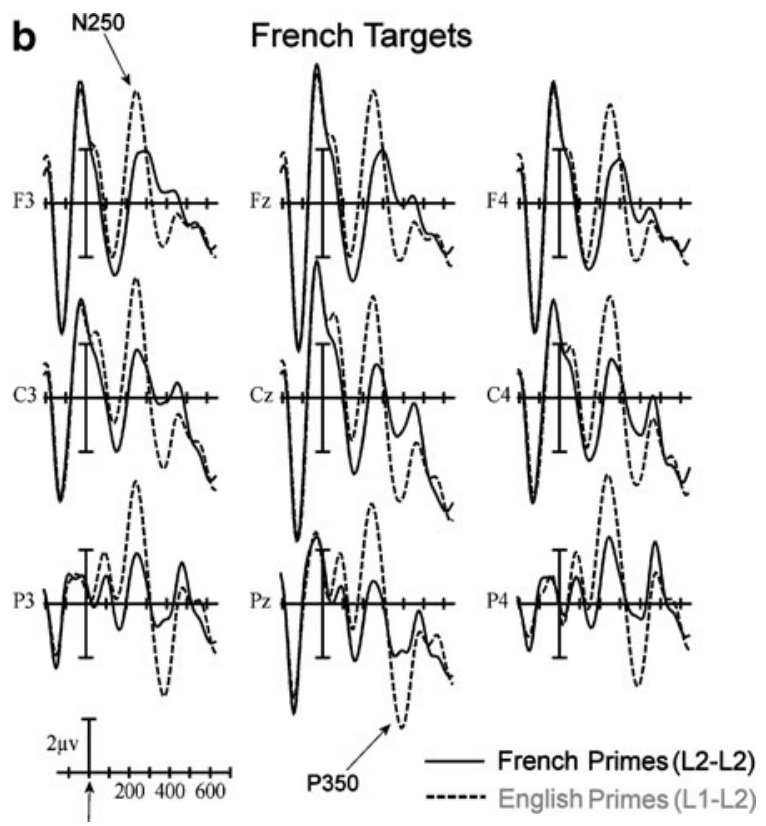

(English) primes (dashed). b Target ERPs in the 100-ms condition of Experiment 1 showing L2 (French) targets preceded by either L2 primes (solid), or L1 (English) primes (dashed) 
0.0158]. However, the pattern of language-switching effects differed as a function of prime duration [prime duration $\times$ language switch interaction, $F(1,38)=6.22, p=.0171$; see Fig. 5a, b). The pattern of more negative-going responses in between-language trials at anterior sites was evident only for the $50-\mathrm{ms}$ prime duration. At the 100 -ms prime duration, between-language trials continued to produce more positivegoing responses than did within-language trials.

\section{Probe task performance}

Participants detected significantly more animal probes in their L1 $(97 \%)$ than in their L2 $(77 \%), F(1,38)=60.3, p=.0001$, but this did not differ as a function of prime duration, $p>.24$. As was expected, participants did not detect any probes in the prime position in the 50-ms prime duration condition but did press to an average of $30 \%$ of prime probes (38\% in L1 and $22 \%$ in L2) in the 100 -ms prime duration condition. False alarms to nonprobe stimuli were fewer than $1 \%$.

\section{Discussion}

The ERP signature of language-switching effects in the Chauncey et al. (2008) study was an increased negativity to target words following a language switch, as compared with the no-switch condition. In the Chauncey et al. study, this pattern was evident mostly in the N250 time window for L2 targets and the N400 time window for L1 targets. Experiment 1 tested a group of more proficient bilingual participants in exactly the same conditions as those our previous study. We found the same early effect of language switching in the N250 time window for L2 targets, as well as the later effect with L1 targets. Furthermore, an analysis of ERPs in a time window lying in between the N250 and the N400 (referred to here as the P350) revealed a large reversed switch effect for L2 targets, with switch trials showing more positive-going waveforms than did nonswitch trials. This pattern of increased positivity in the language switch condition was also evident for L1 targets in the early (N250) time window. Finally, another novel aspect of the results of Experiment 1, as compared with our previous study, is that prime duration interacted with the language-switching effects. Language-switching effects were larger (bigger amplitude differences) at the longer prime duration in Experiment 1, and this influence of prime duration was statistically significant for the L2 targets. Fig. 6 shows the difference waves reflecting the size and the direction of switch effects for each target language and prime duration.

However, one problem in interpreting the languageswitching effects observed by Chauncey et al. (2008) and in

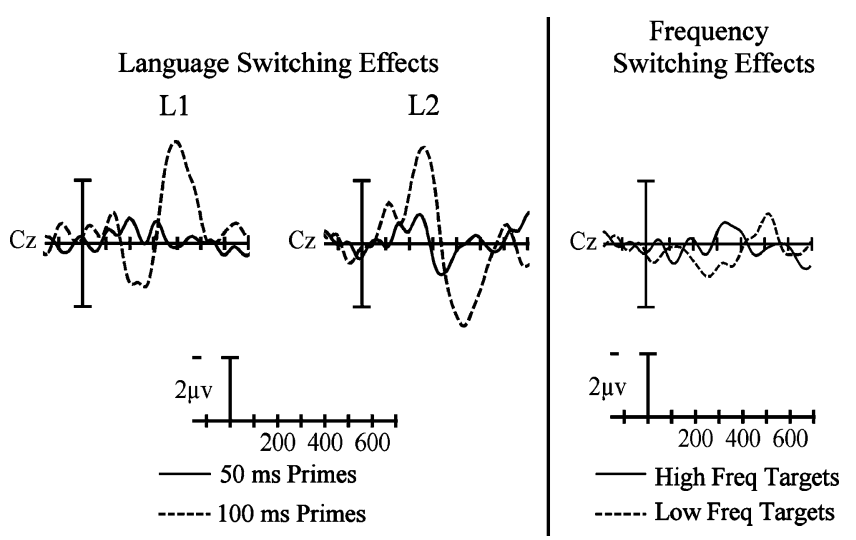

Fig. 6 Difference waves formed by subtracting the voltage values at one representative electrode site $(\mathrm{Cz})$. On the left, the nonswitch condition is subtracted from the switch condition for L1 and L2 targets at the 50-ms (solid) and 100-ms (dashed) prime durations of Experiment 1. On the right are subtractions of the high-frequency targets when preceded by a low- versus high-frequency prime word (solid) and low-frequency targets when preceded by high- versus lowfrequency primes (dashed) in Experiment 2

Experiment 1 of the present study is that at each time window, the pattern of switch effects differed as a function of the direction of the switch. This means that it might not be a change in language that is driving these effects, but simply the language of the prime stimulus. Midgley, Holcomb, and Grainger (2009) examined the influence of language dominance (L1 vs. L2) on the ERP waveforms generated by single words in bilingual participants. They found an increased negativity for L1 words, as compared with L2 words, that mostly affected N400 amplitude but started to emerge as early as $150 \mathrm{~ms}$ post-stimulus-onset. ${ }^{1}$ Given this finding, it could indeed by the case that the effect of language switching found for L2 targets in the N250 time window might be an effect of prime language, with L1 primes causing the increased negativity during the early phase of target processing. On the other hand, the languageswitching effects found with L1 targets in the N400 time window cannot be due to the effects of prime language per se, since the effects should have gone in the opposite direction, with greater negativities in the nonswitch condition (L1-L1) than in the switch condition (L2-L1).

\section{Experiment 2}

Experiment 2 was designed to examine another possible interpretation of the early increased negativity in the

\footnotetext{
${ }^{1}$ An analysis of language effects in Experiment 1, comparing the L1-L1 condition with the L2-L2 condition, revealed systematically greater negativities for L1 words than for L2 words in both the N250 and N400 time windows.
} 
language switch condition with L2 target words, first reported by Chauncey et al. (2008) and replicated in the present Experiment 1. It could be the case that L1 prime words, as compared with L2 prime words, generate an early interference effect in the processing of L2 target words by virtue of their higher subjective frequency. Experiment 2 therefore examined whether any of the language-switching effects found in Experiment 1 could be attributed to differences in the subjective frequency of L1 and L2 words in our bilingual participants. Twenty of the bilingual participants from Experiment 1 were tested in their L1 (English) with low-frequency and high-frequency target words primed by unrelated low-frequency and highfrequency words. Here we wanted to see whether prime word frequency would modify the ERP waveforms to target words and whether this modulation would differ as a function of target word frequency. If subjective frequency were the source of our language-switching effects, we ought to see an influence of prime word frequency on ERP amplitudes, with the high-frequency-prime-low-frequencytarget condition mimicking the L1-L2 language switch effects, and the low-frequency-prime-high-frequency-target condition mimicking the L2-L1 effects.

\section{Method}

Experiment 2 used the same apparatus, procedures, and task as those in Experiment 1, except that a single prime duration was chosen $(70 \mathrm{~ms})$, lying between the short and long durations used in Experiment 1. Twenty of the participants who took part in Experiment 1 were tested again in Experiment 2.

\section{Stimuli}

Two hundred high-frequency words and 200 low-frequency words were selected (see Table 2 for stimulus characteristics). Prime and target word frequency were crossed in a $2 \times 2$ design with target frequency (high vs. low) and frequency switch (switch vs. nonswitch) as factors, giving the following four conditions: high-frequency primes followed by unrelated low-frequency targets, lowfrequency primes followed by unrelated high-frequency targets, high-frequency primes followed by unrelated highfrequency targets, and low-frequency primes followed by unrelated low-frequency targets. Four lists were constructed in order to place every low-frequency and highfrequency item in each condition across participants. There were 50 items in each condition, for a total of 200 critical trials per list. Trials with low-frequency targets and trials with high-frequency targets were run in separate blocks, thus matching the conditions of the language-switching experiments. All participants received the two blocks, and the order of blocks was counterbalanced. All prime types had an equal mix of parts of speech, and neighborhood density, length, and concreteness were matched across frequency range. Care was taken to ensure that there was minimal form and meaning overlap across primes and targets.

\section{Procedure}

The go/no-go semantic categorization task of Experiment 1 was used again. Participants were asked to press a button whenever they saw an animal word. In each frequency block, $15 \%$ of the trials contained an animal word, half in the prime position and half in the target position. All else was as the same as in Experiment 1.

\section{Results}

The results were analyzed in exactly the same way as in the previous experiment, except that the frequency switch factor now replaced the language switch factor. Target word frequency was therefore blocked just like target language in the previous experiment, and the data were analyzed separately. The same three posttarget windows (200-300, 300-400, and 400-500 ms) were used to analyze the ERP data.

\section{High-frequency targets}

Plotted in Fig. 7 are the ERPs to high-frequency targets preceded by primes that are either also high frequency (solid) or low frequency (dashed). Plotted in Fig. 4b (top row) are the voltage maps derived by subtracting highfrequency targets preceded by low-frequency primes from high-frequency targets preceded by high-frequency primes.

Table 2 Properties of the high-frequency and low-frequency words tested in Experiment 2

\begin{tabular}{lllll}
\hline & Number of Letters $(S D)$ & Sum of Bigram Frequency $(S D)$ & Number of Neighbors $(S D)$ & Log Frequency $(S D)$ \\
\hline LF & $5.4( \pm 0.70)$ & $10651.2( \pm 4343.52)$ & $3.06( \pm 1.59)$ & $0.328( \pm 0.34)$ \\
HF & $5.45( \pm 1.05)$ & $10017.93( \pm 4794.63)$ & $3.05( \pm 2.71)$ & $2.250( \pm 0.17)$ \\
\hline
\end{tabular}




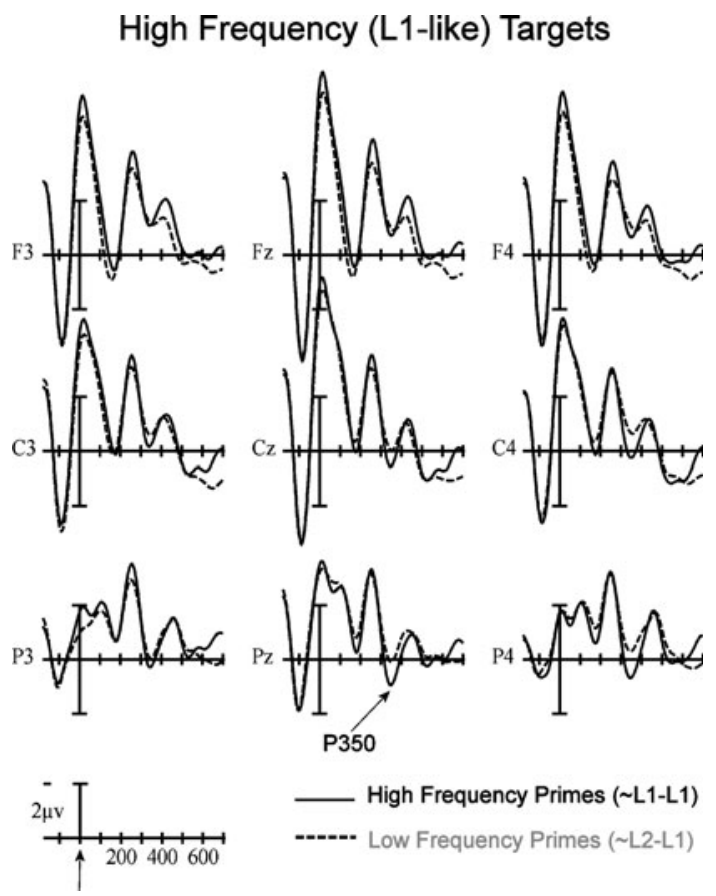

Fig. 7 ERPs to selected sites in Experiment 2, showing highfrequency primes (analogous to L1 words) followed by highfrequency targets (solid) and low-frequency primes (analogous to L2 words) followed by high-frequency targets (dashed)

\section{$200-300 \mathrm{~ms}$}

There were no significant effects in this window.

$300-400 \mathrm{~ms}$

There were no significant effects in this window.

$400-500 \mathrm{~ms}$

In this window, switch trials were more positive than were nonswitch trials at left-hemisphere sites [frequency switch $\times$ laterality, $F(2,36)=5.006, p=.017]$.

\section{Low-frequency targets}

Plotted in Fig. 8 are the ERPs to low-frequency targets preceded by primes that are either low-frequency (solid) or high-frequency (dashed) words. Plotted in Fig. 4b (bottom row) are the voltage maps derived by subtracting lowfrequency targets preceded by high-frequency primes from low-frequency targets preceded by low-frequency primes.

\section{$200-300 \mathrm{~ms}$}

There was a main effect of frequency switch, with nonswitch targets producing more negative-going waves than did switch targets, $F(1,19)=6.15, p=.023$.

\section{$300-400 \mathrm{~ms}$}

In this time window, switch trials were more positive than nonswitch trials [main effect of frequency switch, $F(1,19)=$ $4.57, \mathrm{p}=.046$ ), and there was a trend for this effect to be larger at midline and right-hemisphere sites (switch $\times$ laterality interaction, $F(2,38)=2.81, p=.073$.

400-500 ms

Switch trials were more positive than nonswitch trials, particularly at posterior sites [frequency switch $\times$ anteriorposterior, $F(2,38)=4.64, p=.016]$.

\section{Probe task performance}

Participants detected $97 \%$ of the probes in the target position and $19 \%$ in the prime position. False alarms to nonprobe stimuli were fewer than $1 \%$.

\section{Discussion}

The critical result of Experiment 2 is that the N250 frequency-switching effect with low-frequency targets went in exactly the opposite direction to the language-switching effect with L2 targets seen in Experiment 1. Whereas an increased negativity on switch trials was seen in the N250 component in Experiment 1 and in Chauncey et al. (2008),

\section{Low Frequency (L2-like) Targets}
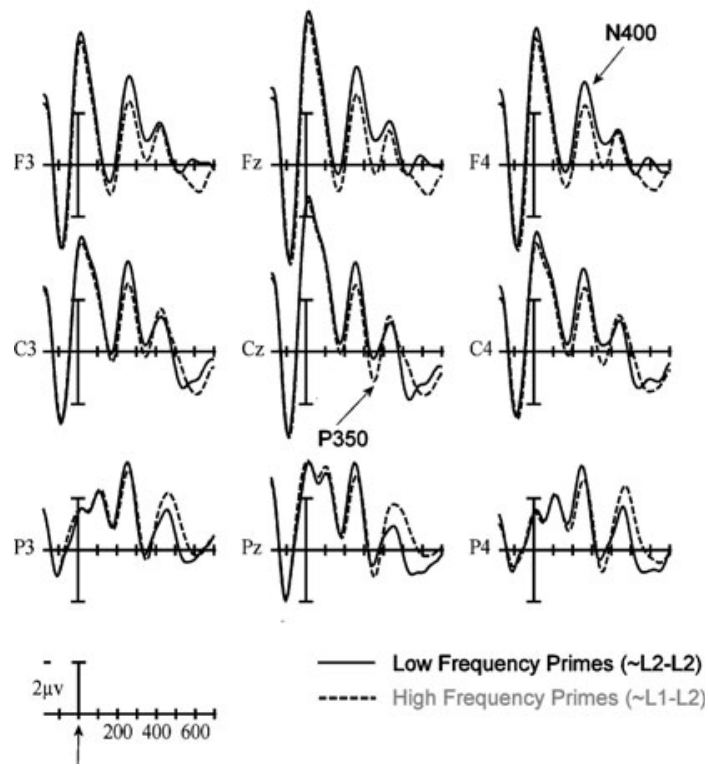

Fig. 8 ERPs to selected sites in Experiment 2, showing lowfrequency primes (analogous to L2 words) followed by lowfrequency targets (solid) and high-frequency primes (analogous to L1 words) followed by low-frequency targets (dashed) 
more positive-going waveforms were seen following a frequency switch in Experiment 2. Therefore, our languageswitching effect in the L1-L2 direction on the N250 component is likely not to be due to the higher subjective frequency of L1 primes, as compared with L2 primes. In the General discussion section, we examine an alternative account of these early L1-L2 switch effects as effects of prime language, rather than prime frequency. With respect to this possibility, it is important to note that the results of Experiment 2 show that the late effect of L2-L1 switches seen in Experiment 1 cannot be explained as a frequencyswitching effect. Although high-frequency targets showed little influence of prime word frequency, the effect on the N400 component was in the opposite direction to the analogous effect of language switching, with frequency switching producing increased positivity, rather than negativity. Finally, the results of Experiment 2 did reveal one effect that appears to have commonalities with the language-switching effects seen in Experiment 1. That is the effect on the P350 component, which was robust for low-frequency targets and in the same direction as that for the analogous L2 targets in Experiment 1.

\section{General discussion}

Experiment 1 in the present study provided a replication of the language-switching effects reported in an earlier study (Chauncey et al., 2008). Here, we tested a group of participants with a higher level of proficiency in their L2 and found that this did not change the overall pattern of switch effects. Just as in our previous study, L2 targets continued to show increased negativity in the N250 component when following L1 (switch) primes, as compared with L2 (nonswitch) primes. The same pattern of increased negativity on switch trials was seen with L1 targets in later time windows, corresponding to the N400 component. Experiment 2 examined whether the early effect seen with L2 targets could be due to the higher subjective frequency of L1 primes, as compared with L2 primes. The results of this experiment showed that a withinlanguage frequency-switching manipulation did not generate the same pattern of effects as that found with language switching. However, one ERP component, lying in between the N250 and N400 and referred to as the P350, was similarly affected by language switching and frequency switching (see Fig. 4).

In the introduction we contrasted two accounts of language-switching costs: the BIA model (Grainger \& Dijkstra, 1992; van Heuven et al., 1998) and the IC model (Green, 1998). According to Green's IC model, task schemas play a central role in generating the languageswitching effects observed in laboratory tasks. Changing languages is akin to changing tasks, such that following a language switch, there is a cost in processing, because the task control mechanism was set to operate for the incorrect language. It is not obvious how this mechanism could account for the fast-acting language-switching effects found with brief prime exposures reported by Chauncey et al. (2008) and replicated in the present study. Since masking minimizes extralexical processing of the masked prime stimulus, if these executive control procedures were the only source of language-switching costs, we would not have expected to see language-switching costs in our masked-priming experiments. Of course, this does not exclude the possibility that task schemas play a role in generating the language-switching effects found with other paradigms, where endogenous (goal-driven) rather than exogenous (stimulus-driven) effects predominate (see Grainger et al., in press, for further discussion of this distinction between exogenous and endogenous languageswitching effects).

With parsimony in mind, we would argue that the results of the present study are more in line with the predictions of the original BIA model (Grainger \& Dijkstra, 1992; van Heuven et al., 1998), according to which languageswitching effects are the result of automatic top-down modulation of activation of lexical representations as a function of language. Prime words rapidly activate the corresponding language node, which then inhibits the representations of all words in the irrelevant language (van Heuven et al., 1998). When the prime and target are from the same language, there is no influence of prime language node activation on the processing of the target word. However, when the prime is from a different language, the target word representation receives inhibition from the language node activated by the prime word, hence influencing ongoing target word processing.

One way of saving task schemas as a general mechanism underlying language-switching effects would be to explain the fast-acting language-switching effects found by Chauncey et al. (2008) and in Experiment 1 of the present study as being driven by factors other than a change in language. In the Discussion section for Experiment 1, we entertained the possibility that it was the language of the prime, as opposed to a change in language across primes and targets, that was driving the different patterns of ERPs. On the basis of the language effects reported by Midgley et al. (2009) and an analysis of comparable effects in Experiment 1, switch effects could be due to L1 primes generating a persisting negativity during L2 target word processing, as compared with L2 primes. This remains a viable explanation for the L1-L2 switch effects seen in the N250 time window in Experiment 1. Similarly, the earliest effects of L2-L1 switches can also be explained as an effect of prime language, rather than a language switch effect, since the 
switch (L2-L1) condition generated reduced negativity, as compared with the nonswitch (L1-L1) condition. However, the later effects of L2-L1 switches reported by Chauncey et al. (2008) and replicated in Experiment 1 cannot be explained by this mechanism, since L2 primes in the switch condition (L2-L1) generated greater negativities than did L1 primes in the nonswitch (L1-L1 condition).

Pursuing alternative interpretations of these languageswitching effects, Experiment 2 of the present study examined whether or not these fast-acting languageswitching effects could, in fact, be driven by lexical frequency. With respect to the discussion above of the possible role of prime language alone in driving early language-switching effects, the key result of Experiment 2 is that frequency-switching effects with high-frequency targets do not show the same pattern as the languageswitching effects seen with L1 targets in the N400 component. These L2-L1 switch effects in the N400 time window are therefore the only effects that can be unequivocally attributed to a change in language across prime and target, as opposed to a direct influence of prime language or prime word frequency. It is therefore possible that fast-acting exogenous language-switching effects are limited to L1 target words, contrary to the predictions of the BIA model, according to which the effects should be stronger with L2 targets. In the BIA model, the faster processing of L1 words would enable a faster acting influence of these primes on the processing of $\mathrm{L} 2$ targets. L1 prime words automatically activate the L1 language node, which then spreads inhibition to all L2 lexical representations. Primes in L2 would take longer to activate the L2 language node-hence, the delayed influence of language switching in the L2-L1 direction. The BIA model therefore predicted stronger language-switching effects that are visible earlier in the ERP waveforms when targets are L2 words, as compared with L1 words.

Clearly, more research is necessary in order to establish whether the early language-switching effects seen with L2 targets in Chauncey et al. (2008) and the present study are, indeed, being driven by the change in language across prime and target. In any case, it is not an unreasonable possibility that language switch effects might be more restricted in the L1-L2 direction than from L2-L1. Indeed, from a developmental perspective, late learners of an L2 need to acquire the capacity to inhibit L1 representations during the processing of L2 words (see Grainger et al., in press, for a specific proposal along these lines). Indeed, language-switching effects in language production follow exactly this pattern (e.g., Meuter \& Allport, 1999). However, the pattern of language-switching effects found in language production need not be the same as that in language comprehension, since production involves goaldriven (endogenous) regulation of irrelevant language activity (I want to speak in language A; therefore, I inhibit lexical representations in language B), whereas comprehension likely involves both endogenous (the next word is most likely going to be a word in language A) and exogenous influences on the activity of the irrelevant language. Future research needs to establish whether the exogenous influences revealed by our fast masked-priming paradigm are indeed restricted to switches from L2 to L1.

Finally, the present study revealed a systematic influence of prime language and prime frequency on the amplitude of a positive-going component lying between the N250 and N400 - referred to here as the P350. This component was more pronounced (more positive-going) following primes in L1, as compared with primes in L2, and the same pattern was found in Experiment 2 for high-frequency primes, as compared with low-frequency primes. Although the P350 effect was always stronger with L2 targets than with L1 targets and with low-frequency targets than with highfrequency targets, the same general pattern was also seen with L1 targets and high-frequency targets (note that this means that the switch effects went in opposite directions as a function of target language/frequency; see Fig. 4). In the light of these results, it is possible that the P350 component reflects a spillover effect of the difficulty of prime stimulus processing during target processing, that arises independently of any relation between prime and target stimuli. Future research is clearly required to identify the functional significance of this specific ERP component.

\section{Conclusions}

The present study provided a direct replication of the fastacting language-switching effects reported in a previous study (Chauncey et al., 2008), while testing participants that were more proficient in their second language. In Experiment 1, switching from an L1 prime word to L2 targets caused an increase in amplitude of the N250 component, as compared with nonswitch L2 trials. This increased negativity on switch trials was also seen with L1 targets in the N400 component. Experiment 2 allowed us to rule out a subjective frequency account of these languageswitching effects by showing that analogous frequency switches within the L1 produced effects that went in the opposite direction. Nevertheless, it is possible that the early language-switching effects from L1 to L2 are, in fact, being driven by a greater negativity generated by L1 prime words than by L2 prime words, independently of a change in language between prime and target. This could not, however, be the case for the switch effects from L2 to L1 seen in the N400. Finally, a pattern of reversed languageswitching effects was also found in Experiment 1, with nonswitch trials generating greater negativity than switch 
trials in the P350 component. The results of Experiment 2 suggest that these particular effects are, in fact, driven by prime word frequency.

Acknowledgments This research was supported by NIH Grants HD043251 and HD25889, awarded to Philip Holcomb and Jonathan Grainger. J.G. was also supported by ERC Advanced Grant 230313. Correspondence concerning this article should be addressed to Jonathan Grainger, Laboratoire de Psychologie Cognitive, Université de Provence, 3 place Victor Hugo, Marseille, France (jonathan. grainger@univ-provence.fr).

\section{References}

Alvarez, R. P., Holcomb, P. J., \& Grainger, J. (2003). Accessing word meaning in two languages: An event-related brain potential study of beginning bilinguals. Brain and Language, 87, 290-304.

Chauncey, K., Grainger, J., \& Holcomb, P. J. (2008). Code-switching effects in bilingual word recognition: A masked priming study with ERPs. Brain and Language, 105, 161-174.

Costa, A., \& Santesteban, M. (2004). Lexical access in bilingual speech production: Evidence from language-switching in highly proficient bilinguals and L2 learners. Journal of Memory and Language, 50, 491-511.

Dalrymple-Alford, E. C. (1985). Language-switching during bilingual reading. British Journal of Psychology, 76, 111-122.

Dijkstra, T., \& van Heuven, W. J. B. (2002). The architecture of the bilingual word recognition system: From identification to decision. Bilingualism: Language \& Cognition, 5, 175-197.

Finkbeiner, M., Gollan, T. H., \& Caramazza, A. (2006). Lexical access in bilingual speakers: what's the (hard) problem? Bilingualism: Language and Cognition, 9, 153-166.

Grainger, J., \& Beauvillain, C. (1987). Language blocking and lexical access in bilinguals. The Quarterly Journal of Experimental Psychology, 39A, 295-319.

Grainger, J., \& Dijkstra, T. (1992). On the representation and use of language information in bilinguals. In R. J. Harris (Ed.), Cognitive processing in bilinguals. Amsterdam: North-Holland.

Grainger, J., \& Holcomb, P. J. (2009). Watching the word go by: On the time-course of component processes in visual word recognition. Language and Linguistics Compass, 3, 128-156.

Grainger, J., Midgely, K., \& Holcomb, P.J. (2010). Re-thinking the bilingual interactive-activation model from a developmental perspective (BIA-d). In M. Kail \& M. Hickman (Eds.), Language Acquisition across linguistic and cognitive systems. John Benjamins

Green, D. W. (1998). Mental control of the bilingual lexico-semantic system. Bilingualism: Language \& Cognition, 1, 67-81.

Holcomb, P. J., \& Grainger, J. (2006). On the time course of visual word recognition: An event-related potential investigation using masked repetition priming. Journal of Cognitive Neuroscience, $18,1631-1643$.

Holcomb, P. J., \& Grainger, J. (2007). Exploring the temporal dynamics of visual word recognition in the masked repetition priming paradigm using event-related potentials. Brain Research, 1180, 39-58.

Kroll, J. F., \& Stewart, E. (1994). Category interference in translation and picture naming: Evidence for asymmetric connections between bilingual memory representations. Journal of Memory and Language, 33, 149-174.

Macnamara, J., \& Kushnir, S. L. (1972). Linguistic independence of bilinguals: The input switch. Journal of Verbal Learning and Verbal Behavior, 10, 480-487.

Meuter, R. F. I., \& Allport, A. (1999). Bilingual language-switching in naming: Asymmetrical costs of language selection. Journal of Memory and Language, 40, 25-40.

Midgley, K. J., Holcomb, P. J., \& Grainger, J. (2009). Language effects in second language learners and proficient bilinguals investigated with event-related potentials. Journal of Neurolinguistics, 22, 281-300.

Moreno, E. M., Federmeier, K. D., \& Kutas, M. (2002). Switching languages, switching palabras (words): An electrophysiological study of code switching. Brain and Language, 80, 188207.

Orfanidou, E., \& Sumner, P. (2005). Language-switching and the effects of orthographic specificity and response repetition. Memory \& Cognition, 33, 355-369.

Soares, C., \& Grosjean, F. (1984). Bilinguals in a monolingual and a bilingual speech mode: The effect of lexical access. Memory \& Cognition, 12, 380-386.

Thomas, M. S. C., \& Allport, A. (2000). Language-switching costs in bilingual visual word recognition. Journal of Memory and Language, 43, 44-66.

van Heuven, W. J. B., Dijkstra, T., \& Grainger, J. (1998). Orthographic neighborhood effects in bilingual word recognition. Journal of Memory and Language, 39, 458-483.

von Studnitz, R. E., \& Green, D. W. (1997). Lexical decision and language-switching. International Journal of Bilingualism, 1, 324. 